\title{
EXTRACTION OF PROTONS ELASTICALLY SCATTERED BY W AND CU TARGETS OF THE IHEP ACCELERATOR. THE FIRST RESULTS.
}

\author{
A. Asseev, Yu. Karshev, A. Maksimov, A. Minchenko, G. Voronin \\ IHEP, Protvino, Moscow Region, 142281, Russia
}

\begin{abstract}
Extraction of the $70 \mathrm{GeV}$ protons has been realized with the aid of the internal targets installed before the septum-magnets of the slow extraction system. Having undergone elastic scattering in the target and a following deflection into the first septum-magnet aperture, protons are extracted from the accelerator. The targets of $\mathrm{W}$ and $\mathrm{Cu}$ were used. As distinct from the regime of simultaneous extraction of protons with the use of targets generating the secondary particles (the material of $\mathrm{Be}, \mathrm{C}$ ) the intensity of more than 3E11 protons per cycle was obtained. It is by 5-10 times higher of the working intensity of the accelerated proton beam extracted simultaneously with the beam of secondary particles.
\end{abstract}

\section{INTRODUCTION}

On a direct extraction of protons scattered by internaltarget was reported in [1]. Particles scattered by angles $~ 5-6 \mathrm{mrad}$ were extracted towards channel 8 . Intensity of $6 \cdot 10^{7}$ at $10^{12}$ of primary protons interacting with a target per cycle (ppc) was registered.

Next step was the extraction of protons scattered by internal targets by much smaller angles $(\sim 1 \mathrm{mrad}[2])$. It was required by the Institute program of physics researche in the frame of which it was necessary to get $10^{7}-10^{11} \mathrm{ppc}$ inaccessible for experiments earlier. Intensity of $3 \cdot 10^{10} \mathrm{ppc}$ was obtained at the FODS setup when $\sim 6 \cdot 10^{11}$ of primary protons interacted with a target installed before the first septum-magnet of the slow extraction system in straight section (SS) 18 [2].

Later (see, for example, [3]-[6]) extraction of primary protons simultaneously with secondary particles for various experiments was realized. Scattering was due to targets of light materials (mainly $\mathrm{Be}$ ) generating secondaries for channels $2(14), 4,18,5 \mathrm{~N}$ etc. Intensity of a proton beam in such mode, for example, for the Tagged Neutrino Facility (TNF) setup was $\sim 3-5 \cdot 10^{10} \mathrm{ppc}[6]$. At the present experiment for $10^{12}$ of protons interacting with the target the intensity of an extracted beam $\geq 3 \cdot 10^{11} \mathrm{ppc}$ was obtained. Increase of an extracted intensity is expected when the certain conditions to be reported below are realized.

\section{SCHEME OF THE EXPERIMENT}

A part of the A-70 perimeter (blocks 20-30) is presented in fig. 1 where a disposition of septum-magnets of SS 24 and 26 is shown. The azimuth of scattering targets is marked as $\mathrm{T}$ there. The local distortions of closed orbit (bumps) used for steering of a circulating beam towards the target (1), for displacement of a beam to a septum of the SM-24 and facilitation for particles to get the apertures of SM-24 and SM-26 (2) and the trajectory of the extracted proton beam (3) are shown also.

There is an azimuthal shift $\sim 0.5$ radian between the scattering target and SM-24. It plays the important role providing extraction of a part of the particles undergone scattering just after interaction. For evaluation we will consider that particles before scattering had zero amplitude. The growth of the amplitude of betatron oscillations of a particle at the azimuth of the SM-24after interaction with a target is expressed as[7]:

$$
\Delta A_{r, z}=\frac{\left|\varphi_{T}\right| \cdot\left|\varphi_{S M}\right|}{V_{0}} \cdot \Phi_{r, z}
$$

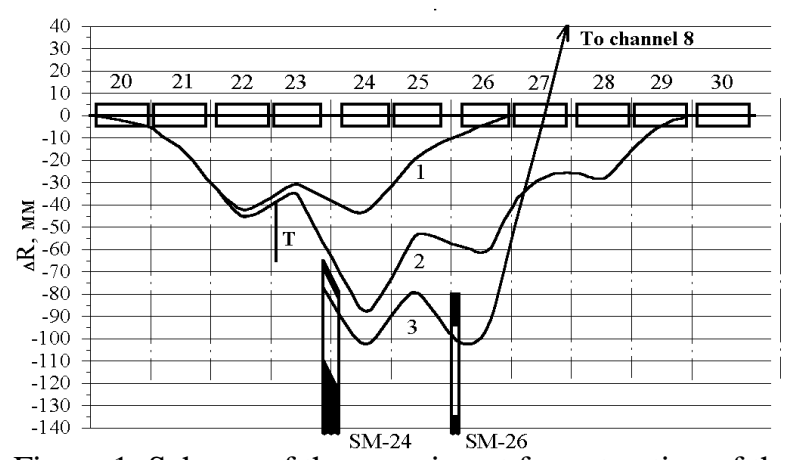

Figure 1: Scheme of the experiment for extraction of the elastically scattered protons.

where: $V_{0}=1 \mathrm{~m} ;\left|\varphi_{T}\right|,\left|\varphi_{S M}\right|$ are modules of the Flo quet functions at azimuths of the target and SM-24 respectively; $\Phi_{r, z}$ is the horizontal or vertical projection of a r.m.s. angle of multiple scattering. From [8]:

$$
\Phi_{r, z}=\frac{E_{s}}{p \beta c} \cdot \sqrt{\frac{L_{T}}{L_{R}}}
$$

where: $E_{s}=15.0 \mathrm{MeV} ; \quad p$ and $\beta c$ are the momentum and velocity of an incident particle; $L_{T}, L_{R}$ are thickness of the target and radiation length of its material. In our case $L_{T}=3 \mathrm{~cm}$.

Displacement of a particle at the SM-24 azimuth due to a single scattering in the target is expressed by :

$$
\Delta X_{r, z}=\Delta A_{r, z} \cdot \sin \left(\psi_{S M}-\psi_{T}\right)
$$

where $\psi_{S M}, \psi_{T}$ are the arguments of the appropriate Floquet functions .

Evaluations show that the horizontal displacement of a particle at the SM-24 azimuth at the first turn can be respectively (for $\mathrm{W}$ and $\mathrm{Cu}$ targets):

$\Delta X_{r W}=6.96 \mathrm{~mm}$ and $\Delta X_{r C u}=3.42 \mathrm{~mm}$. At the physical thickness of a septum $\sim 2.0 \mathrm{~mm}$ it is seen that already at 
the first turn after intersection the target particles can get an aperture of the first deflector.

One can note that before to be lost due to elastic nuclear interaction with the target of $\mathrm{W}$, a proton can cross the target twice, as $\lambda_{T}^{W}=5.71 \mathrm{~cm}$. It gives an increase of a scattering angle and, respectively, growth of amplitude of oscillation by $\sqrt{2}$, i.e. $\Delta A_{r W 2}=9.84 \mathrm{~mm}$. In the case of $\mathrm{Cu}$ target $\left(\lambda_{T}^{C u}=9.55 \mathrm{~cm}\right)$ protons can cross a target more than 3 times. As a result: $\Delta A_{r C u 3}=5.92 \mathrm{~mm}$.

It means, that even for non-optimal position of a beam relatively to a septum of the deflector scattered particles will be thrown into its aperture. The situation is more favorable if a change of particle phase due to circulation and ionization energy loss are taken into account.

\section{EXPERIMENTAL RESULTS}

In our case the result of the experiment was optimal at a gap between a beam and a septum of the SM-24 (input coordinate is $R=-65 \mathrm{~mm}) \sim 1-2 \mathrm{~mm}$ before steering it towards a target. Dependence of intensity of the extracted beam (in relative units) versus a coordinate of a scattering target is shown in fig.2.

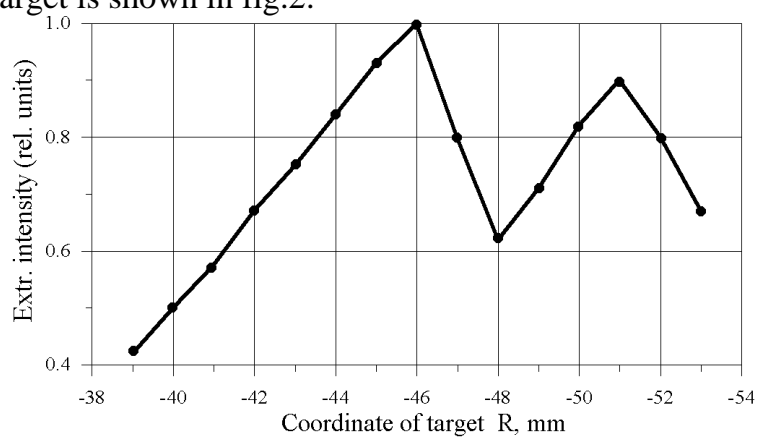

Figure 2: Dependence of the extracted beam intensity versus a coordinate of a scattering target.

Analysis of fig. 2 shows:

1. Intensity of the extracted beam is due to the mutual position of a beam and a septum.

2. Maximum of the extracted intensity corresponds to the target coordinate $R=-46 \mathrm{~mm}$. At the closed orbit bump used it corresponds to the gap between a beam and septum of $2 \mathrm{~mm}$.

3. The "downfall" of the intensity curve means that around $\mathrm{R}=-48 \mathrm{~mm}$ a target gets a "shadow" of a septum. Some particles of the circulating beam and particles scattered by small angles are lost.

The extracted beam profiles measured at the accelerator exit (at SS-30) show that the size of a beam is $\sim 12-15$ $\mathrm{mm}$. At an effective thickness of the septum $\sim 3 \mathrm{~mm}$ and a gap between a beam and septum $\sim 2 \mathrm{~mm}$ one can see that the amplitude of scattered particles is $\sim 17-20 \mathrm{~mm}$. It agrees well with evaluations of tab.1.

It is necessary to point out that replacement of $\mathrm{W}$ target by $\mathrm{Cu}$ does not change its optimal coordinate but makes narrower the profile of the extracted beam. Intensity gets higher by about 1.5 times $\left(\sim 2.5 \cdot 10^{11}\right.$ comparing to $\sim 1.6 \cdot 10^{11} \mathrm{ppc}$ in the case of $\mathrm{W}$ target). The explanation of the effect is given in the next section. Shortening of the targets thickness up to $10 \mathrm{~mm}(\mathrm{~W})$ and $20 \mathrm{~mm}(\mathrm{Cu})$ allowed one to get the extracted intensity $\geq 3 \cdot 10^{11} \mathrm{ppc}$.

\section{DISCUSSION OF RESULTS}

It follows from results of the experiment that one can provide the necessary growth of betatron amplitudes and extract particles undergone multiple Coulomb scattering to the experimental setups with an aid of the septum-magnets of a slow extraction system. If at the given parameters of a target (material, traversed thickness etc.) a probability of inelastic nuclear interaction for a single pass of the target is small ( $\leq$ of several percents) it means that extraction of a beam of rather high intensity is possible. The losses of protons at narrow points of the accelerator vacuum chamber should be minimized.

It follows from (3) that at the fixed azimuth of the target the displacement of a particle at the azimuth of the septum-magnet depends on a shift of phase $\left(\psi_{S M}-\psi_{T}\right)$ which increases by $2 \pi Q$ after each turn of incident particle. Here $Q$ is a frequency of betatron oscillation of beam particles. In our case the working point corresponded to Qr/Qz=9.73/9.70. Respectively, displacement of particles at the azimuth of the SM-24 will be changed by a magnitude and sign from a turn to turn. Values for the cases of $\mathrm{W}$ and $\mathrm{Cu}$ targets are given in tab.1. Dependence from number of intersections of the target by a particle is included also.

Table 1. Horizontal displacements of a particle versus a number of intersections of the target.

\begin{tabular}{|c|c|c|c|c|c|}
\hline \multirow{2}{*}{$\begin{array}{c}\mathrm{N} \\
\text { of } \\
\text { turns }\end{array}$} & \multicolumn{4}{|c|}{ Number of target intercections } \\
\cline { 2 - 6 } & $\mathrm{W}: \Delta X_{r W}, \mathrm{~mm}$ & \multicolumn{3}{c|}{$\mathrm{Cu}: \Delta X_{r C u}, \mathrm{~mm}$} \\
\hline & 1 & 2 & 1 & 2 & 3 \\
\hline 0 & 6.96 & 9.84 & 3.42 & 4.84 & 5.92 \\
\hline 1 & -13.42 & -18.98 & -6.60 & -9.33 & -11.43 \\
\hline 2 & -3.60 & -5.09 & -1.77 & -2.50 & -3.06 \\
\hline 3 & 14.31 & 20.38 & 7.05 & 9.97 & 12.21 \\
\hline
\end{tabular}

It is seen that in our scheme for particles scattered by the "negative" angle the first turn (number 0) and fourth one (number 3), when the change of coordinates have a necessary sign and their magnitude exceeds a thickness of the septum, are favourable. For the particles undergone scattering by a "positive" angle the second turn (number 1) when the modulus of the coordinates are close to the maximum value achieved at the fourth turn is favourable.

So, notwithstanding of a sign of a scattering angle, a particle can get such a growth of an amplitude of oscillation that at the certain turn after scattering it will be thrown into the aperture of the first magnetic deflector (the SM-24 in our case) and extracted from the accelerator. Next (second, third etc.) intersections of the target by a particle provide a further growth of betatron 
amplitudes and a throw of particles into the aperture of the first deflector even in the case when the gap between a beam and a septum before scattering is large (see fig.2). Naturally, the particle should not be lost yet due to nuclear interaction in the target (elastic or inelastic). Intensity of the extracted beam at significant clearances between a beam and a septum is, respectively, less...Tab.2. helps to clear a situation with the case of extraction a beam of about 1.5 times higher intensity with the aid of $\mathrm{Cu}$ target.

Tab.2. Vertical displacements of particle versus a number of interactions with the target.

\begin{tabular}{|c|c|c|c|c|c|}
\hline \multirow{2}{*}{$\begin{array}{c}\mathrm{N} \\
\text { of } \\
\text { turns }\end{array}$} & \multicolumn{5}{|c|}{ Number of scattering } \\
\cline { 2 - 6 } & $\mathrm{W}: \Delta X_{z W}, \mathrm{~mm}$ & \multicolumn{3}{|c|}{$\mathrm{Cu}: \Delta X_{z C u}, \mathrm{~mm}$} \\
\cline { 2 - 6 } & 1 & 2 & 1 & 2 & 3 \\
\hline 0 & 4.93 & 6.97 & 2.43 & 3.44 & 4.21 \\
\hline 1 & -20.08 & -28.39 & -9.88 & -13.97 & -17.11 \\
\hline 2 & 7.46 & 10.55 & 3.67 & 5.19 & 6.36 \\
\hline 3 & 15.15 & 21.42 & 7.60 & 10.75 & 13.16 \\
\hline
\end{tabular}

Since the acts of scattering of particles both in horizontal and vertical planes are statistically independent and the r.m.s. values of scattering angles are iqual [8], the vertical coordinates of particles after scattering are determined by a phase difference between a target and septum-magnet and the phase shift due to circulation (see formula 3). One can see from tab.2 that in the case of $\mathrm{W}$ target the particles, independently of a sign of scattering, at the second turn have a displacement more than $20 \mathrm{~mm}$. Since the vertical aperture of the SM-24 does not exceed $\pm 15 \mathrm{~mm}$, a noticeable part of particles should be lost on vertical walls of the first deflector. The effect of "trimming" will be more noticeable if the vertical distortions of the beam orbit are taken into account. In case of $\mathrm{Cu}$ target a situation is more favorable: displacement of a particle exceeds a size of the SM-24 vertical half-aperture after the third intersection of the target only when the probability of the elastic nuclear scattering is high.

A few words about influence of ionization energy losses. At intersection of a target the momentum $p_{0}$ of an incident proton is changed by a value $\Delta p$. Respectively a radial position of the closed orbit at the azimuth of a septum-magnet will be changed by:

$$
\Delta r_{S M}=-\psi_{S M}^{\Delta p} \cdot \frac{\Delta p}{p_{0}}
$$

Here $\psi_{S M}^{\Delta p}$ is a value of dispersing function at the septum-magnet azimuth. It is easy to get that the orbit displacement at the azimuth of SM-24 contributes to betatron amplitude of incident particle $\sim 7 \mathrm{~mm}$ (for 2 intersections of $\mathrm{W}$ target) and $\geq 5 \mathrm{~mm}$ (for 3 intersections of $\mathrm{Cu}$ ). Together with multiple Coulomb scattering this effect helps for particles to be thrown in the SM-24 aperture at the first turn after scattering.

\section{CONCLUSION}

The obtained results allow one to hope that in our scheme one can get intensity of the extracted beam $\sim 5 \cdot 10^{11}$ ppc when $10^{12}$ of particles interact with the target. Only losses of particles on vertical walls of the septum-magnet apertures should be minimized. In particular for the thickness of $\mathrm{W}$ target $\sim 7 \mathrm{~mm}$ and $\sim 15 \mathrm{~mm}$ for $\mathrm{Cu}$ the r.m.s. scattering angles $\Phi_{r, z}$ are respectively 0.28 and $0.22 \mathrm{mrad}$. After first 2-3 intersections of a target amplitude growth of particles will not exceed the vertical aperture limits of the SM-24.

Further increase of extracted intensity is possible if one ensures a phase shift between the target and the first deflector of $\sim \pi / 2$.

- In the existing scheme a growth of a betatron amplitude which depends on the product of modules of the Floquet functions is maximal if the target is placed in the focusing block 20. Deflection of a particle $\Delta X_{r}$ at the azimuth of SM-24 at the first turn after scattering will noticeably exceed a thickness of the septum (for $\mathrm{W} \sim 7.7$ $\mathrm{mm}$, for $\mathrm{Cu} \sim 6.0 \mathrm{~mm}$ ). The vertical losses of particles will be minimized.

- One can use the SM-18 with a septum thickness of $\sim 0.7 \mathrm{~mm}$. Scattering target is installed in magnetic block 14. Losses of particles due to a decrease of both the septum thickness and $\left|\varphi_{z}\right|$ will be significantly lowered.

- The electrostatic deflector of the SS106 with the wires of $\sim 0.15 \mathrm{~mm}$ can be used. According to evaluations, the beam of intensity up to $10^{12}$ ppc can be extracted with a good time structure.

In the last two cases there is a possibility of a stable extraction of a proton beam of intensity $\sim 5 \cdot 10^{11}$ or more for certain experiments (for example, to search the rare decays of $\mathrm{K}-$ mesons at the TNF setup, to research the polarizing processes at the setup FODS-2) simultaneously with extraction of secondary particles for experiments at other channels. It is the most preferable mode of work providing the stable and qualitative conditions for all 4-5 simultaneously made experiments.

\section{REFERENCES}

[1] A.V.Alexeev et al., IHEP 79-98, Serpukhov, 1979.

[2] A.A.Asseev et al., IHEP 85-23,Serpukhov , 1985.

[3] A.A.Asseev et al., JTP, 1990, v.60, N9, p. 70.

[4] A.A. Asseev et al., Proceed. of the 2-nd European PAC, Nice, 1990, v.2, p.1604.

[5] A.A. Asseev et al., Proceed. of the 3-d European PAC, Berlin, 1992, v.2, p. 1486.

[6] A.A. Asseev et al., Proceed. of the 5-th European PAC, Barcelona, 1996, v.3, p. 2409.

[7] A.A.Kolomensky, A.N.Lebedev. The theory of the cycle accelerators. ) ., P) , 1962.

[8] D.Perkins. Introduction to high energy physics. ) ., Energoatomizdat, 1991. 